Cleveland Clinic, OH. One fourth of focal seizure patients had more than a $50 \%$ reduction in seizure frequency after 3 months and 12 months, and 6 patients had more than $90 \%$ control. Generalized seizures responded more favorably, with $46 \%$ having a $50 \%$ reduction at 3 months and $42 \%$ at 12 months. Differences were not significant. Outcome tended to be better in patients under 12 years of age. (Maydell BV, Wyllie E, Akhtar N et al. Efficacy of the ketogenic diet in focal versus generalized seizures. Pediatr Neurol September 2001;25:208-212). (Respond: Dr Elaine Wyllie, Head, Section of Pediatric Epilepsy, The Cleveland Clinic Foundation, Department of Neurology, Desk S 51, 9500 Euclid Ave, Cleveland, $\mathrm{OH} 44195)$.

COMMENT. The ketogenic diet is a treatment option in young children with medically intractable focal seizures when surgery is not advised. Like antiepileptic drugs, however, the ketogenic diet may be accompanied by adverse events and treatment must be carefully monitored.

Adverse effects of the ketogenic diet. Wheless JW discusses the possibie aciverse events during initiation and maintenance of the ketogenic diet (I Child Neurol Sept 2001;16:633-35). These include the following: a) diet initiation adverse events: dehydration, hypoglycemia, and vomiting; and b) during diet maintenance: poor growth, kidney stones, hyperlipidemia, cardiomyopathy, prolonged QT interval, excessive bruising, optic neuropathy, elevated very-longchain fatty acids, vitamin D deficiency, osteomalacia, trace mineral deficiencies, constipation, exacerbation of gastroesophageal reflux disease. Diseases that may be aggravated by the ketogenic diet include: porphyria, pyruvate carboxylase deficiency, carnitine deficiency, fatty acid oxidation defects, and mitochondrial disorders. Parents expecting that a diet treatment is "natural" and relatively innocuous should be counseled regarding risks. Deaths related to the ketogenic diet have been reported in 3 children (cardiac arrest, pneumonia, and acute hemorrhagic pancreatitis), and serious adverse events in 5 children included hypoproteinemia, hemolytic anemia, thrombocytopenia with bleeding, Fanconi's renal tubular acidosis, and elevated liver enzymes. (Ped Neur Briefs July 2001;15:50-52).

The Hopkins method of introduction, using a period of starvation and maintenance of a $4 / 1$; fat/nonfat ratio, appears to be more conducive to adverse events than the Mayo Clinic method that omits a starvation period and employs lower ratios. (Millichap JG. Progress in Pediatric Neurology I, PNB Publishers, 1991;pp84-88).

\title{
ANTIEPILEPTIC DRUGS IN AUTISTIC SPECTRUM DISORDERS
}

Published data on the use of antiepileptic drugs (AED) in the treatment of autism (ASD) and associated affective disorders and epilepsy have been reviewed at the Department of Neurology, Miami Children's Hospital, FL. A total of 10 case reports or open-label studies included 2 adults and 29 children, ages 22 months to 14 years. Mental retardation was present in 21 of the 31 patients, epilepsy (generalized and complex partial) in 18, and EEG abnormalities in the absence of clinical seizures in 6. Affective symptoms (manic or depressive episodes) occurred in 7. In one report of 13 patients, MRI and SPECT were abnormal in $50 \%$ and $100 \%$, respectively, without specific details noted. Antiepileptic drugs (valproic acid (VPA) in 4, lamotrigine (LMG) in 13, and carbamazepine (CBZ) in 1) were used to control seizures in all 18 patients with epilepsy and ASD, and in 5 of 6 patients with EEG abnormalities without seizures. They were used as mood stabilizers in 7 children with affective symptoms without seizures. VPA was effective in control of seizures and EEG abnormalities in all 8 children treated, CBZ was effective in 3 
of 4 chjildren, and LMG was effective in 6 of 13 patients. Improved communication skills were reported in all 8 receiving VPA, 8 of 13 on LMG, and 2 of 3 treated with CBZ. Improvements in communication and socialization skills were correlated with control of seizures or EEG abnormalities. Improvement in affective symptoms in all 7 patients with mood disorders following therapy with VPA and CBZ was associated with control of seizures and EEG epileptiform abnormalities. Placebocontrolled, double-blind studies are required to investigate the role of AED in the management of affective disorders, ASD, with or without seizures and abnormal EEG. Autism, affective disorders, and epilepsy are frequently comorbid and may share common neuroanatomic and neurochemical neural circuits. (Martino AD, Tuchman RF. Antiepileptic drugs: affective use in autism spectrum disorders. Pediatr Neurol September 2001;25:199-207). (Respond: Dr Roberto F Tuchman, Director, Dan Marino Center, Department of Neurology, Miami Children's Hospital, 2900 South Commerce Parkway, Weston, FL. 33331).

COMMENT. Uncontrolled studies suggest that antiepileptic drugs may have a role in the management of affective disorders and autistic spectrum disorder, with or without an associated epilepsy or abnormal EEG. The frequent use of AED in the management of behavior disorders in children with epileptiform EEGs in the absence of clinical seizures indicates the urgent need for controlled studies. The authors have provided an excellent review of the available data on this important problem and the coexistence of autism, epilepsy, and affective disorders.

\section{MOVEMENT DISORDERS}

\section{NEUROBEHAVIORAL OUTCOME IN OPSOCLONUS-MA SYNDROME}

The relationship between long-term neurobehavioral outcome, MRI findings, and late anti-neuronal antibodies was examined in 11 children with neuroblastoma and opsoclonus-myoclonus-ataxia syndrome (OMA) followed for a mean of 7.6 years at the University of California San Francisco, CA. Seven (64\%) exhibited only mild neurologic deficits, $2(18 \%)$ had severe deficits, and $2(18 \%)$ had none. Four (36\%) had severe cognitive and behavioral deficiences, while 6 (55\%) were average and 1 (9\%) moderately below average. Brain MRI showed cerebellar atrophy in 5 of 5 tested, whereas antineuronal activity was not detected in the sera of 10 children examined at follow-up. (Hayward $\mathrm{K}$, Jeremy RJ, Jenkins $S$ et al. Long-term neurobehavioral outcomes in children with neuroblastoma and opsoclonus-myoclonus-ataxia syndrome: relationship to MRI findings and antineuronal antibodies. L Pediatr October 20901;139:552-559). (Reprints: Katherine K Matthay MD, Department of Pediatrics, UCSF Medical Center, 505 Parnassus Ave, M-647, San Francisco, CA 94143).

COMMENT. Neurologic examination alone is an insufficient indicator of overall function in long-term follow-up of children with neuroblastoma and OMA syndrome. Cognitive tests are the most sensitive indicator of neurobehavioral deficits. Despite residual neurologic abnormalities, some patients have average neurobehavioral function, with continued improvement over time. Late cerebellar atrophy is a common finding and may be related to neuropsychological sequelae; it did not appear to be related to steroid use. The absence of antineuronal activity (ANA) at follow-up negates any role for ANA in continuing neurologic damage in OMA. Behavioral, fine-motor, and academic performance, in addition to neurologic and MRI examinations, should be monitored in the follow-up of children with OMA. 